\title{
Il est temps de resserrer les rangs
}

\section{Ludwig T. Heuss}

Prof. Dr, président du conseil d'administration des EMH Editions médicales suisses

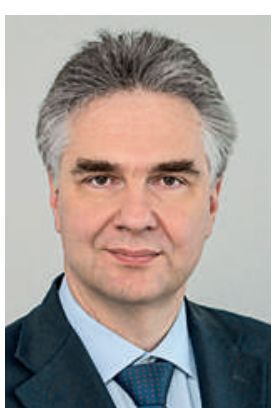

Cela ne vous a pas échappé: l'édition du Bulletin des médecins suisses que vous avez entre les mains est aussi une nouvelle revue de formation continue. Les couleurs et la mise en page rappellent peut-être des choses connues, mais le fond est nouveau: une production commune des rédactions du Swiss Medical Forum (SMF) et de la Revue Médicale Suisse (RMS). Les deux parents se présentent fièrement à la une et la revue n'a vraiment pas à avoir honte de ses origines. Sous la houlette avisée du Prof. Gérard Waeber de Lausanne, les deux magazines de formation médicale continue leaders en Suisse se sont réunis et ont produit un numéro commun. Pour l'instant, il n'y en a qu'un, mais il sera suivi d'un second à l'automne. C'est un premier pas dans la bonne direction.

\section{Une coopération au-delà des frontières} linguistiques.

$\mathrm{Au}$ travers de sa décision de novembre 2011, la Chambre médicale a exigé une telle coopération audelà des frontières linguistiques et il n'y a pas de raison de ne pas l'approfondir. Au contraire. Le Swiss Medical Forum et la Revue Médicale Suisse ont tous deux été créés par des maisons d'édition suisses indépendantes appartenant à des organisations professionnelles, autrement dit par des médecins, pour les médecins. Les Editions médicales suisses EMH sont une maison d'édition majoritairement détenue par la FMH qui, conformément aux buts de notre association professionnelle, promeut par le biais de trois produits clés la communication indépendante, au service de la qualité, sur des thèmes de politique professionnelle, de formation postgraduée et continue, ainsi que scientifiques. La société coopérative Médecine et Hygiène, qui publie la Revue Médicale Suisse, est liée à la Société Médicale de la Suisse Romande. Lors d'une telle collaboration, le fait que les deux entreprises sont animées par le même esprit devient perceptible - pour nous, mais aussi pour vous, lectrices et lecteurs de ce numéro. Mais leurs cultures se complètent également.

On peut s'en étonner en raison du caractère international de la recherche médicale, de l'EbM et d'internet, mais la langue, la société et le système de santé marquent toujours la médecine d'une empreinte locale. Celle-ci est importante car de sa somme découle la pratique des actes médicaux à laquelle nous nous identifions et dont nous nous sentons proches. Un coup d'œil par-delà du «Röstigraben» permet de percevoir ce qui, entre Genève et le Romanshorn, entre Bâle et Chiasso, est devenu l'essence de ce qui fait la médecine "suisse» autochtone. On peut spontanément supposer qu'elle est caractérisée par la lucidité et le pragmatisme, l'attachement aux faits, la défiance à l'encontre des hiérarchies et un sens aigu du service.

Au-delà de la barrière linguistique, il convient de faire progresser une compréhension commune de ce qui doit être préservé et encouragé. Un coup d'œil à l'environnement souligne également l'importance et le symbolisme d'une telle collaboration. Les médias médicaux, de même que l'ensemble de la branche, sont depuis des années et de plus en plus soumis à une pression économique considérable. Cela crée des dépendances et un risque d'accepter les compromis, de céder à la pression permanente d'influence illégitime sur les contenus éditoriaux, comme on le constate aisément en passant au crible certaines revues du marché.

\section{Les deux entreprises sont animées par le} même esprit.

Grâce à la FMH et à l'abonnement de base lié à la cotisation de membre, le Swiss Medical Forum et la Revue Médicale Suisse sont en mesure de résister à cette pression. C'est la FMH, le corps médical suisse, qui garantit notre indépendance. En tant qu'éditeurs, nous suivons la médecine suisse à la trace, nous tentons de défendre nos couleurs, y compris face à des géants internationaux de la presse, à la taille sans comparaison avec la nôtre. "Collaborer est un succès» écrivent les rédacteurs impliqués dans leur éditorial. Car plus que jamais, il importe de faire preuve d'esprit pionnier et de flexibilité et de resserrer les rangs. Aux Editions médicales suisses EMH, nous nous réjouissons d'intensifier encore cette coopération. 